\title{
Magnetizing Current, Harmonic Content and Power Factor as the Indicators of Transformer Core Saturation
}

\author{
Ismail Daut, Syafruddin Hasan, and Soib Taib
}

\begin{abstract}
The paper proposes a measurement method for obtaining the parameters needed to calculate a reliable indicator of the state of transformer core saturation. The major nonlinear effects in transformer core are saturation, eddy current and hysteresis. Saturation is the predominant effect in power transformers, followed by eddy current and hysteresis effects. In saturation, a transformer core act as a source of current generating harmonics, some of which will flow directly toward the primary and secondary windings. The method is based on magnetization current, harmonic content and power factor evaluated of the transformer no-load current with high magnetic flux density subjected. The investigation is done on single phase model core type transformer. The transformer is connected to variable voltage variable frequency (VVVF) source and the investigation is done on various flux densities. The results show that the magnetization current and harmonic content increase significantly when high magnetic flux densities are injected and vice versa with power factor that decrease sharply. These phenomena can be used as the indication of transformer core saturation.
\end{abstract}

Index Terms-Harmonic content, no-load current, power factor, saturation, transformer

\section{NOMENCLATURES}

$B$ magnetic flux density

$B_{r} \quad$ remanence magnetic flux density

$B_{s} \quad$ saturation magnetic flux density

$C R G O$ Cold-Rolled Grain Oriented

$H$ magnetic field intensity

$H_{c}$ coercivity magnetic field intensity

$H_{p k}$ peak value of magnetic field intensity

$H_{s}$ saturation magnetic field intensity

IEC International Electrotechnical Commission

IEEE Institute of Electrical and Electronic Engineers

$I_{o} \quad$ magnetizing current

$I_{r m s}$ rms value of all the current harmonic component

$I_{l, r m s}$ rms value of fundamental current

$I_{h, r m s}$ rms value of current harmonic component of order $\mathrm{h}$

M4 commercial name for grain oriented silicon steel sheet's grade

Manuscript received December 16, 2012; revised February 18, 2013.This work was supported in part by Centre of Excellence for Renewable Energy and School of Electrical Systems Engineering, Universiti Malaysia Perlis (UniMAP) for the technical and financial support as well.

I. Daut is with Centre of Excellent for Renewable Energy, School of Electrical Systems Engineering, Universiti Malaysia Perlis (e-mail: ismail.daut@unimap.edu.my).

S. Hasan is with Centre of Excellent for Renewable Energy, School of Electrical Systems Engineering, Universiti Malaysia Perlis and Fakultas Teknik, Univ.Sumatera Utara (USU) Medan Indonesia (e-mail: syafruddin@unimap.edu.my).

S. Taib is with the School of Electrical \& Electronic Engineering, Universiti Sains Malaysia (e-mail: soibtaib@eng.usm.my).
$P \quad$ power

$P F$ total (true) power factor

$P F_{\text {disp }}$ displacement power factor

$P F_{\text {dist }}$ distribution power factor

THDi percent total current harmonic distortion

$V_{\text {lrms }}$ rms value of fundamental voltage

$\mu \quad$ permeability of core material

\section{INTRODUCTION}

The magnetic circuit is one of the most important active parts of transformer. It consists of laminated iron core and carries flux linked to windings. Energy is transferred from one electrical circuit to another through the magnetic field carried by the core. The iron core provides a low reluctance path to the magnetic flux thereby reducing magnetizing current.

The performances of transformers are also constrained by the magnetic flux limitations of the core. For ferromagnetic core transformers, we must be mindful of the saturation limits of the core. Remember that ferromagnetic materials cannot support infinite magnetic flux densities: they tend to "saturate" at a certain level (dictated by the material and core dimensions), meaning that further increases in magnetic field force $(\mathrm{mmf})$ do not result in proportional increases in magnetic field flux.

Choosing the right lamination material for a particular application is an important power transformer design step, since lamination properties have a direct link to the losses power or efficiency of the transformer. Currently, important lamination properties (core loss and permeability) to be used for assessment are presented at 50 or $60 \mathrm{~Hz}, 1.0 / 1.5 \mathrm{~T}$ sinusoidal. Electromagnetic designers have to decide on the material based on this single operating point, although under practical working conditions, some transformers operate at flux density levels and frequencies beyond this point. It has been shown that choosing the lamination material that based on the lamination performance at one operating point only is not enough [1]. Designers of transformers have, however, never had data available on the specific iron losses of the materials they use for alternating flux densities higher than of the saturation value. The usual methods of measurement, applied to specimens of the material, fail completely at higher flux densities than because the power factor becomes very small and the magnetizing current very high [2].The major nonlinear effects in transformer iron core are saturation, eddy current and hysteresis. Saturation is the predominant effect in power transformers, followed by eddy current and hysteresis effects [3]. The iron core of transformers and other electromagnetic devices is laminated so that at $50 \mathrm{~Hz}$ or 60 $\mathrm{Hz}$ (or, in general, at power frequency) skin effect is 
insignificant. Due to saturation however, harmonics are generated in either the flux or the magnetic field intensity or in both, and for these the spatial distribution is not uniform.

In saturation, a transformer core act as a source of current generating harmonics, some of which will flow directly toward the primary and secondary windings. On the basis of this observation, the paper proposes a measurement method for obtaining the parameters needed to calculate a reliable indictor of the state of saturation of transformers [4]. The method is based on harmonic content evaluated of the transformer no-load current with high magnetic flux density subjected. The results show that the harmonic content increase significantly when high magnetic flux density injected.

Nowadays, loads of transformers are widely perturbed with harmonics. Harmonic components cause the transformer losses to increase and thus, transformer may fail to operate [5]

\section{HARMONIC PHENOMENA AND CORE SATURATION}

\section{A. Harmonic Phenomena}

The non linear magnetic characteristics of a transformer core are needed to predict distortion of its primary current. One of the methods of estimating harmonic load content is Percent Total Harmonic Distortion (\%THD). The \%THD can be defined in two different ways, as a percentage of the fundamental component (the IEEE definition of THD) or as a percentage of the rms (used by the Canadian Standards Association and the IEC) [7]. In this paper we use the later one.

The percentage current total harmonic distortion is

$$
T H D i=\frac{\sqrt{\sum_{h=2}^{\infty} I_{h, r m s}^{2}}}{I_{r m s}} \times 100 \%
$$

where $I_{h, r m s}$ is the amplitude of the harmonic component of order h (i.e., the $h t h$ harmonic) and $I_{r m s}$ is the rms values of all the harmonics) that can be represented as

$$
I_{r m s}=\sqrt{\sum_{h=1}^{\infty} I_{h, r m s}^{2}}
$$

The total power factor,

$$
P F=\frac{P}{V_{1, r m s} I_{1, r m s} \sqrt{1+\left({ }^{T H D_{i}} / 100 \%\right)^{2}}}
$$

The total power factor is sometime called the true power factor. As shown in equation (3) the total power factor is the product two components. The first component is called the displacement power factor:

$$
P F_{\text {disp }}=\frac{P}{V_{1, r m s} I_{1, r m s}}
$$

The second component of the total power factor is called distortion power factor and results from the harmonic component of the current:

$$
P F_{\text {dist }}=\frac{1}{\sqrt{1+\left(\frac{T H D_{i}}{100 \%}\right)}}
$$

If the voltage also has harmonic components, then the distortion power factor would be the product of two terms like the right side of equation (6), one for voltage and one for current.

$$
P F_{\text {dist }}=\frac{1}{\sqrt{1+\left(\frac{T H D_{i}}{100 \%}\right)}} \times \frac{1}{\sqrt{1+\left(\frac{T H D_{v}}{100 \%}\right)}}
$$

However, we normally expect the voltage distortion to be very low compared to the current distortion.

\section{B. Core Saturation}

A typical hysteresis loop of a soft magnetic material is shown in Fig.1. When a high magnetizing force is encountered, a point is reached where further increase in, $\mathrm{H}$, does not cause useful increase in, B. This point is known as the saturation point of that material. The saturation flux density, $\mathrm{B}_{\mathrm{s}}$ and the required magnetizing force, $\mathrm{H}_{\mathrm{s}}$, to saturate the core are shown with dashed lines[8].

In Fig. 1, the hysteresis loop clearly shows the remanence flux density, Br. The remanence flux is the polarized flux remaining in the core after the excitation has been removed The magnetizing force, $-\mathrm{H}_{\mathrm{c}}$, is called coercivity. It is the amount of magnetizing force required to bring the remanence flux density back to zero.

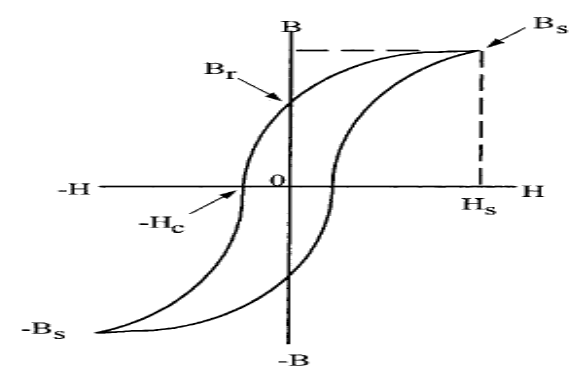

Fig. 1. Typical B-H curve of soft magnetic material.

\section{Permeability}

The permeability of a magnetic material is a measure of the ease in magnetizing the material. Permeability, $\mu$ is the ratio of the flux density, $\mathrm{B}$, to the magnetizing force, $\mathrm{H}$.

$$
\mu=\frac{B}{H}
$$

The relationship between $\mathrm{B}$ and $\mathrm{H}$ is not linear, as shown in the hysteresis loop in Fig.1. Then, it is evident that the ratio, $\mathrm{B} / \mathrm{H}$, (permeability), also varies.

The variation of permeability with flux density, $B$, is shown in Fig. 2. Also, it shows the flux density at which the permeability is at a maximum.

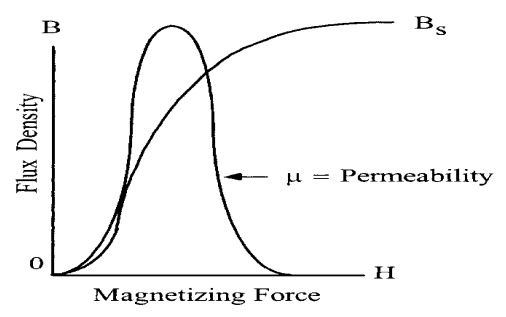

Fig. 2. Variation in permeability with $\mathrm{B}$ and $\mathrm{H}$. 
Silicon steel was one of the first alloys to be used in transformers and inductors. It has been greatly improved over the years and is probably, pound for pound, the most, widely used magnetic material. One of the drawbacks in using steel in the early years was, as the material became older, the losses would increase. With the addition of silicon to the steel, the advantages were twofold: it increased the electrical resistivity, therefore reducing the eddy current losses, and it also improved the material's stability with age. Silicon steel offers high saturation flux density, a relatively good permeability at high flux density, and a moderate loss at audio frequency. One of the important improvements made to the silicon steel was in the process called cold-rolled, grain-oriented,

By definition, saturation occurs when the peak exciting current $(\mathrm{B})$ is twice the average exciting current $(\mathrm{A})$ as shown in Fig. 3. Analytically, this means that [8]:

$$
H_{p k}=2 H_{s}
$$

Refer to Fig. 3, saturation occurs when $\mathrm{B}=2 \mathrm{~A}$

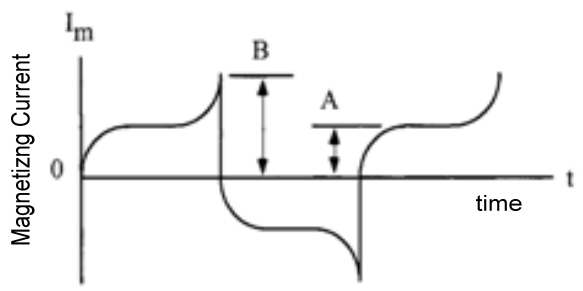

Fig. 3. Defining the excitation current

\section{Model of EXPERIMENTAL SET-UP}

The transformer test has the specification as single-phase power transformer with primary winding of 220 turns and secondary of 220 turns respectively. The dimension of the core is $508 \mathrm{~mm} \times 306 \mathrm{~mm}$ with yoke and limb $100 \mathrm{~mm}$ width. The core is assembled from 0.27 millimeter thickness lamination of M4 grain-oriented silicon iron (CRGO) that comprises of 17 layers and $5 \mathrm{~mm}$ corner overlap length configuration (shown in Fig. 4).

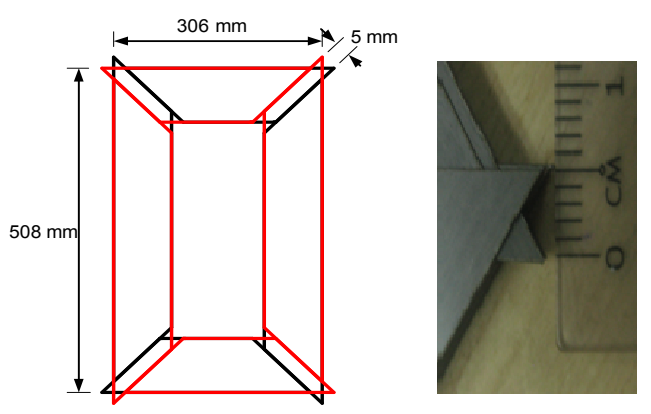

Fig. 4. Transformer core configuration

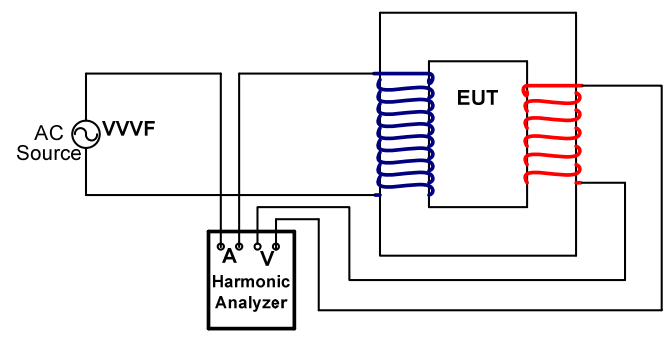

Fig. 5. Arrangement of experimental set-up
The arrangement of equipment set-up which used in this research work is as shown in Fig. 5. The input current to harmonic analyzer (PM 300) is connected to ac variable voltage variable frequency supply and its voltage is come from the secondary side of transformer test (transformer turn ratio 1:1). The experiment was conducted with different flux density by varying the input voltage and the frequency maintain constant.

\section{RESUlT}

The sample of current and voltage waveforms for $50 \mathrm{~Hz}$ of frequency supply with magnetic flux density of 1.5 Tesla and 1.9 Tesla are shown in Fig. 6 (a) and Fig. 6 (b).

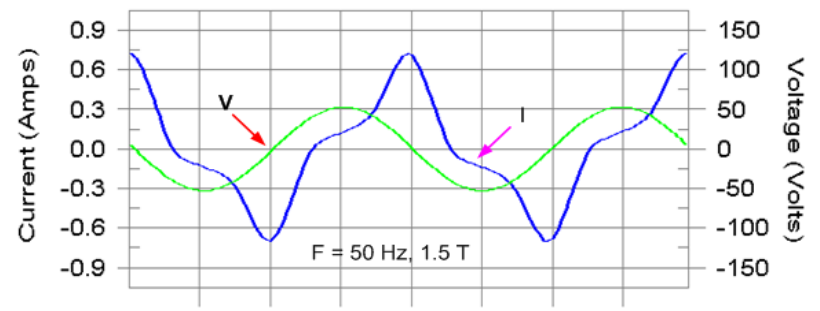

(a) $\mathrm{B}=1.5$ Tesla

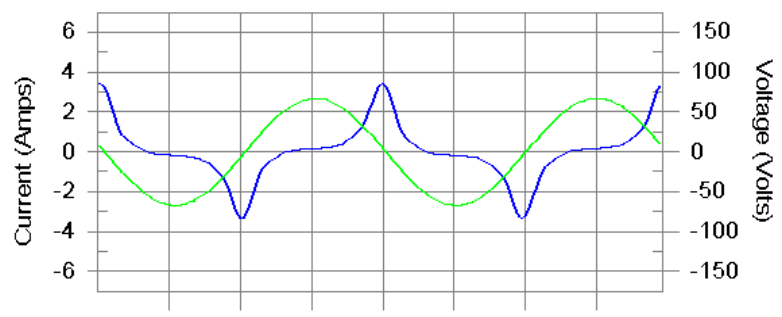

(b) $\mathrm{B}=1.9$ Tesla

Fig. 6. Current and voltage waveforms

The harmonic phenomena in term of current total harmonic distortion and magnetization current (Io) of single-phase transformer at various magnetic flux densities for frequency $50 \mathrm{~Hz}$ is displayed in Table.

TABLE I: MAGNETIZING CURRENT, \%ThDi AND PF MEASURED AT VARIOUS MAGNETIC FLUX DENSITIES

\begin{tabular}{cccc}
\hline \hline B [T] & Io [A] & THD [\%] & PF \\
\hline 1.0 & 0.146 & 16.92 & 0.621 \\
1.1 & 0.172 & 21.23 & 0.587 \\
1.2 & 0.211 & 25.15 & 0.525 \\
1.3 & 0.258 & 28.58 & 0.457 \\
1.4 & 0.317 & 31.62 & 0.395 \\
1.5 & 0.388 & 33.44 & 0.342 \\
1.6 & 0.475 & 37.33 & 0.274 \\
1.7 & 0.594 & 44.48 & 0.174 \\
1.8 & 0.827 & 56.38 & 0.108 \\
1.9 & 1.385 & 64.83 & 0.092 \\
\hline 2.0 & 2.486 & 75.66 & 0.054 \\
\hline \hline
\end{tabular}

The related magnetization current and percent total harmonic distortion of magnetization current (\%THDi) and the respective magnetic flux densities for frequency $50 \mathrm{~Hz}$ are depicted as shown in Fig. 7 and Fig. 8. Meanwhile, the curve of power factor versus magnetic flux densities is displayed in Fig. 9. 


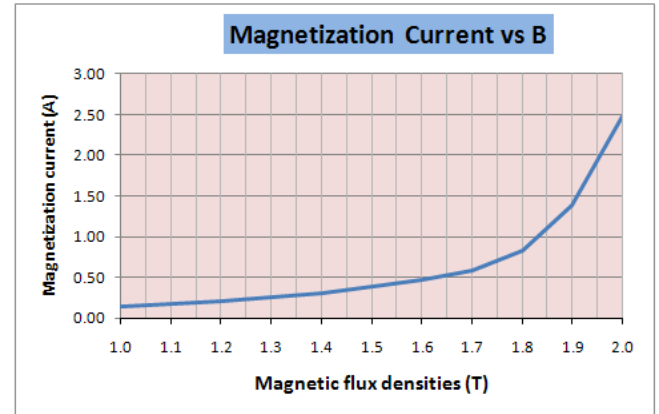

Fig. 7. Magnetization current versus magnetic flux density

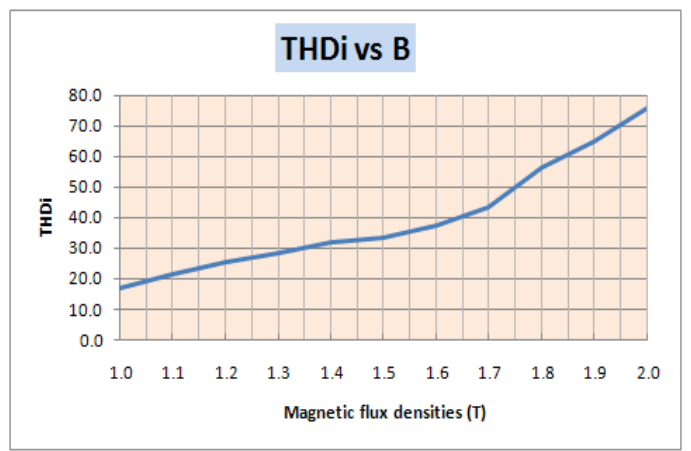

Fig. 8. \%THD versus magnetic flux density

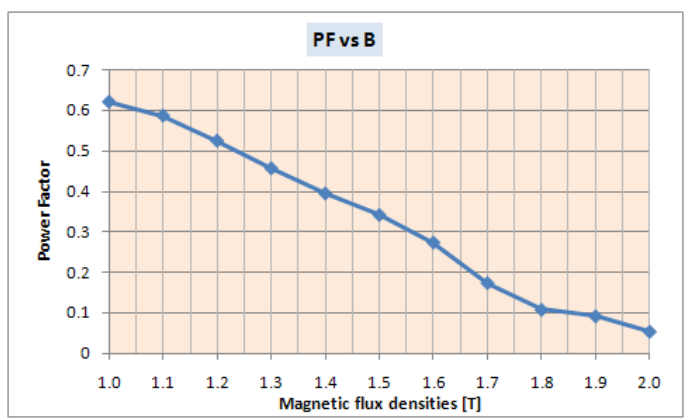

Fig. 9. Power Factor versus magnetic flux density

\section{DISCUSSION}

Fig. 6(a) and (b) show the waveforms of supply voltage and current with different magnetic flux density, i.e, $1.5 \mathrm{~T}$ and $1.9 \mathrm{~T}$. Although the applied voltage is sinusoidal, the current waveform is not sinusoidal. It is clear from Fig.7(a) and (b) that the non-sinusoidal current is still symmetrical around zero crossing of voltage waveform.

The magnetizing current (Io) and harmonics phenomena which are evaluated in terms of percent total harmonic distortion of current (THDi) and power factor are shown in Table 1. The excitation (magnetizing) current, Io at magnetic flux density $\mathrm{B}=1.8 \mathrm{~T}$ (i.e., Io $=0.827 \mathrm{~A}$ ) is more than doubled compare to at 1.4 or $1.5 \mathrm{~T}$ (Io $=0.317 \mathrm{~A}$ or 0.388 ). Meanwhile, the \%THDi increased when the transformer is subjected with higher magnetic flux density. The increasing of \% THD is very significant when the magnetic flux densities are changed from 1.7 to $2.0 \mathrm{~T}$. It could be signified that the lamination cores of transformer lead to saturate. Fig. 7 and Fig. 8 also show that the pattern of Io and \%THDi versus magnetic flux density nearly the same as B-H curve of a magnetic material.

As mention in Equation (3), the power factors are inverse proportional to \%THDi. These phenomenon are displayed in Table I or Fig. 9. It is obvious that the power factors are decreasing significantly when the magnetic flux densities are changed from $1.7 \mathrm{~T}$ to $2.0 \mathrm{~T}$. It could be also as indication that the lamination cores of transformer have been lead to saturate.

\section{CONCLUSION}

Test data obtained for to no-load measurement of single-phase transformer was reproduced in the Centre of Excellence for Renewable Energy (CERE), UniMAP.

This paper presented a practical method for the estimation of nonlinear core saturation in term of magnetization current, its harmonics content and power factor. Analysis of the test data indicate the high magnetic flux density contribute more magnetization current, high harmonic content and low power factor rather than at normal magnetic flux density of actual transformer operation (1.4 or 1.5 Tesla).

The pattern of magnetizing current and percentage current total harmonic distortion versus magnetic flux densities are nearly the same as B-H curve of a magnetic material.

Therefore, these phenomenon can be used as indicator to show that at 1.8 Tesla the material of transformer core has been lead to saturation.

\section{REFERENCES}

[1] K. Karsai, D. Kerenyi, and L. Kiss, Large power transformers, Elsevier Publication, Amsterdam, 1987, pp. 41.

[2] E. So, R. Arseneau, and E. Hanique, "No-Load loss Measurements of Power Transformers under Distorted Supply Voltage waveform Conditions," IEEE Transaction on Instrumentation and Measurement, vol. 52, no. 2, April 2003, pp. 429-432.

[3] R. Arseneau, E. So, and E. Hanique, "Measurements and Correction of No-Load Losses of Power Transformers," IEEE Transaction on Instrumentation and Measurement, vol. 54, no. 2, pp. 503-506.

[4] A. H. A. Haj and I. E. Amin, "Factors that Influence Transformer No-Load Current Harmonics," IEEE Transaction on Power Delivery, vol. 15 , no. 1 , January 2000, pp. 163-166.

[5] M. Mirzaie, M. Y. Asrami, S. B. Sadati, and A. S. Akmal, "Impacts of Non-Sinusoidal Load Currents on Distribution Transformer Losses-Part II: Standard Aspects and Experimental Measurement," International Review of Electrical Engineering (IREE) Journal, vol. 6. no. 5, October 2011, pp. 2215-2220.

[6] V. S. Kulkarni and A. S. Khaparde, Transformer Engineering Design and Practice (Marcel Dekker, Inc., New York, 2004)

[7] International Electrotechnical Commission Standards, IEC 76, part one, Power Transformers, $2^{\text {nd }}$ ed., 1993.

[8] S. L.Timothy and D. W. E. William, Electric Power and Controls, Pearson-Prentice Hall, New Jersey, United States of America, 2004.

[9] C. W. T. McLyman, Transformer and Inductor Design Handbook, $3^{\text {rd }}$ Edition, Revised and Expanded, Marcel Dekker. Inc., New York, 2004.

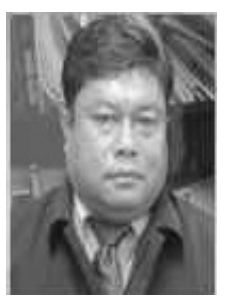

Ismail Daut received his B. Elect.Eng. (Hons) from University of Science Malaysia in 1980 and MSc in Electrical and Electromagnetic Engineering from University of Wales, College of Cardiff, United Kingdom in 1991 and $\mathrm{PhD}$ in Energy Conservation and Power Engineering from University of Wales, College of Cardiff, United Kingdom in 1994. His research interest includes energy conversion, electrical machine design and high voltage. He has authored and co-authored more than 200 technical papers in the national, international journal and conferences.

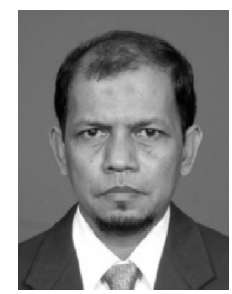

Syafruddin Hasan was born in July 1, 1959 in Pidie, Aceh-Indonesia. He received his BSc from Universitas Sumatera Utara (USU) Medan in 1985 and MSc from Institut Teknologi Bandung (ITB) Bandung-Indonesia, 1993 all in Electrical Engineering. He is a lecture in UniMAP and USU. He is currently PhD student in Universiti Malaysia Perlis (UniMAP). His field of interest includes Electromagnetic Theory, Electrical Machines, Power Electronic Drives and Power Quality. 